\title{
The Oculus Rift virtual colonoscopy: introducing a new technology and initial impressions
}

\author{
David Randall ${ }^{* 1}$, Peter Metherall ${ }^{2}$, Karna Dev Bardhan ${ }^{3}$, Paul Spencer ${ }^{3}$, Richard Gillott ${ }^{3}$, Rebecca de Noronha ${ }^{4}$, John \\ W Fenner ${ }^{5}$ \\ ${ }^{1}$ Medical Physics Group, Department of Cardiovascular Science, The University of Sheffield, Sheffield, UK \\ ${ }^{2} 3 D$ Imaging Laboratory, Sheffield Teaching Hospitals NHS Foundation Trust, Sheffield, UK \\ ${ }^{3}$ The Rotherham NHS Foundation Trust, Rotherham, UK \\ ${ }^{4}$ Medical Imaging and Medical Physics, Sheffield Teaching Hospitals NHS Foundation Trust, Sheffield, UK \\ ${ }^{5}$ INSIGNEO Institute for in silico Medicine, The University of Sheffield, Sheffield, UK
}

Received: November 20, 2015

DOI: $10.5430 / j b g c . v 6 n 1 p 34$
Accepted: December 17, 2015 Online Published: December 22, 2015

URL: http://dx.doi.org/10.5430/jbgc.v6n1p34

\begin{abstract}
Introduction: Virtual colonoscopy has proven to be a reliable, sensitive technique and is now widely adopted in routine clinical practice. We present a novel extension to virtual colonoscopy by incorporating the immersive Oculus Rift virtual reality technology. We describe our initial experience to explore its potential diagnostic benefit. To the best of our knowledge, this is the first Oculus Rift related publication in the radiology field.

Method: A procedure for generating an Oculus Rift virtual colonoscopy (ORVC) has been created. It is derived from a CT image of an air/ $/ \mathrm{CO}_{2}$ inflated colon with contrast labelled faecal matter. The mucosal surface is segmented from its surroundings and subsequently meshed and rendered to produce a 3D surface. The mucosal surface is then imported into the Unity game engine along with the calculated coordinates of its centreline. Within Unity, a virtual reality camera is assigned to travel both outside and inside the colon, aided by controls allocated to an Xbox controller. Two ORVCs were created from differing quality CT data and trialled by two experienced radiologists and a gastroenterologist.

Results: The ORVCs prompted an enthusiastic response from the clinicians. It was considered to offer potential for improved diagnostic power but further development is required if its full capability is to be realised.

Conclusions: A mechanism for examining virtual colonoscopies within the immersive environment of the Oculus Rift has been developed. The resulting ORVCs were trialled by three clinicians with largely positive feedback providing encouragement for pursuing this work further.
\end{abstract}

Key Words: Oculus Rift, Virtual reality, Virtual colonoscopy, CT colonoscopy, Radiology, Diagnostic imaging

\section{INTRODUCTION}

Bowel cancer is the second most common cause of cancer related deaths, with an estimated 16,000 deaths per year in the UK. ${ }^{[1]}$ Optical colonoscopy is the preferred method of colonic examination and is considered the "gold standard".
During the procedure the full length of the colon can be examined in detail with an externally controlled camera inserted into the rectum while also allowing therapeutic procedures (polypectomies - removal of potentially pre-cancerous lesions). Its limitations however are that it has many contraindi-

*Correspondence: David Randall; Email: drandall1 @ sheffield.ac.uk; Address: Medical Physics, IICD, O-Floor, the Royal Hallamshire Hospital, Glossop Rd, Sheffield, South Yorkshire, UK. 
cations and the process is invasive: involving a thorough bowel cleansing, which patients often find unpleasant. ${ }^{[2]}$ Virtual colonoscopy therefore offers an attractive alternative because of its minimally-invasive nature and nominal bowel preparation. A wealth of evidence now offers significant support for the use of virtual colonoscopy as a diagnostic tool $^{[3-6]}$ and as such it has almost entirely replaced barium enemas in the developed world.

The virtual colonoscopy procedure involves an abdominal CT scan, inflation of the colon with air/carbon dioxide and the addition of a contrast medium to distinguish between bowel wall and faecal matter (e.g. gastrografin). The colon is separated (segmented) from the CT image and its inner surface rendered so it can be viewed from inside the lumen of the colon. Virtual colonoscopies are typically viewed and reported on a standard reporting workstation and the camera position/orientation controlled via mouse and keyboard. This paper explores the potential improvements offered to this traditional user interface by the introduction of virtual reality.

The Oculus Rift is a virtual reality headset capable of providing stereoscopic vision and head tracking capabilities [Oculus VR, Irvine, California, USA]. The commercialised version of the Oculus Rift has yet to be released and this paper has experimented with its precursor - the Development Kit 2 (DK2). It has primarily been developed for the gaming industry but has features which could prove useful to the medical field. A handful of publications indicate the Oculus Rift has begun to find applications in medicine ${ }^{[7-12]}$ but as yet none have been applied to diagnostic imaging. Virtual colonoscopy is one medical imaging example where the power of the examination is naturally augmented by the incorporation of stereoscopic vision and head tracking.

An immersive virtual colonoscopy experience has been previously attempted by Mirhosseini et al. in $2014^{[13]}$ using a CAVE (cave automatic virtual environment) - placing the user in a room where the inner surface of the colon is projected onto the surrounding walls. They discuss several aspects of their technique and conclude that it offers potential for improved examination time and accuracy. However, a CAVE system is not practicable for the clinic nor is it financially viable. This paper describes an alternative immersive method for viewing virtual colonoscopies with the Oculus Rift DK2, followed by a discussion of our experiences: highlighting any benefits/downfalls regarding image interpretation.

\section{METHOD}

A total of two Oculus Rift virtual colonoscopies (ORVC) have been produced for viewing by a diagnostician. The following text describes the procedure employed to view a high resolution rendered colon within the immersive environment of the Oculus Rift DK2. An outline of the process used to produce an ORVC is shown in Figure 1.

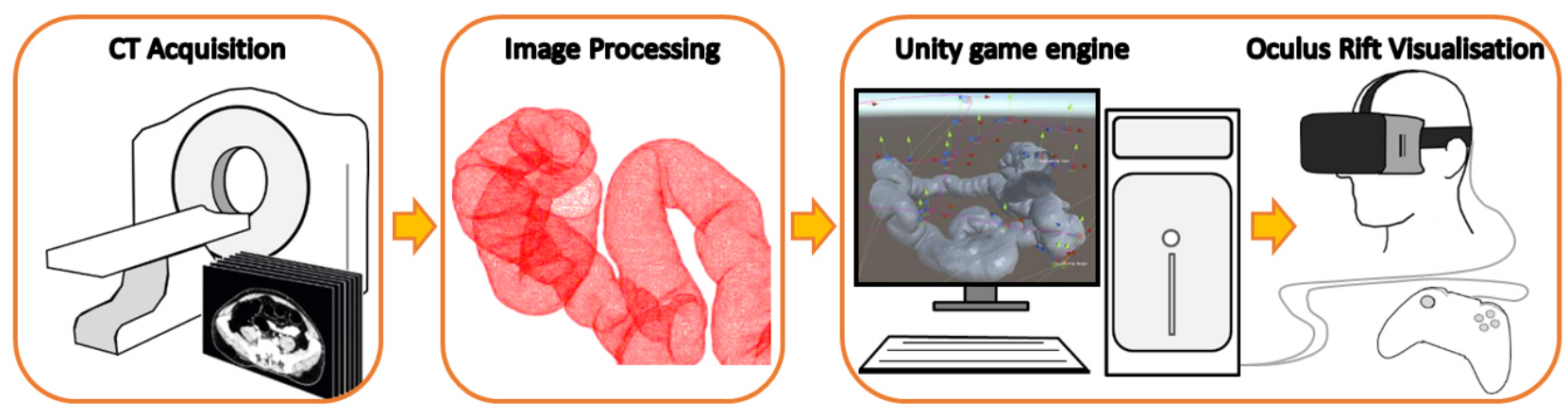

Figure 1. Schematic of the process used to produce an Oculus Rift virtual colonoscopy (ORVC)

\subsection{CT acquisition and image processing}

Demonstration virtual colonoscopy images, freely available for research purposes, were provided by TeraRecon Inc [Foster City, California, USA]. This comprised two studies with differing acquisition parameters in order to assess the technique with varying quality data. The acquisition parameters of the studies are tabulated in Table 1.

Only supine images were examined in this work although the

Published by Sciedu Press same technique is equally valid for prone positions. Images were processed using Mimics Medical 18.0 [Materialise NV, Leuven, Belgium]. The DICOM images were first segmented using threshold and region growing methods. A 3D surface mesh was next generated and optimised to ensure sufficient mesh quality using standard meshing tools available in the software. The ends of the meshed colon were cut to create an open ended vessel structure. The mesh was exported in 
DXF format so that it could be imported directly into the Unity game engine, version 5.1 [Unity Technologies, San Francisco, California, USA].

A centreline analysis was performed on the surface mesh and edited to give a single path through the colon. In the second study obstructed segments of the colon were joined together using a straight line connection between the discrete segments to give a continuous path. The centreline path was exported in an xml format using the associated 3-matic Medical software 10.0 [Materialise NV, Leuven, Belgium].

Table 1. CT acquisition parameters for the two virtual colonoscopy studies

\begin{tabular}{ll}
\hline Study 1 & Study 2 \\
\hline Siemens Sensation 16 & GE Medical Systems LightSpeed Plus \\
$280 \mathrm{~mA}$ & $150 \mathrm{~mA}$ \\
$120 \mathrm{kV}$ & $120 \mathrm{kV}$ \\
Pixel size $0.74 \mathrm{~mm}$ & Pixel size $0.81 \mathrm{~mm}$ \\
Slice increment $0.5 \mathrm{~mm}$ & Slice increment $1.5 \mathrm{~mm}$ \\
Slice thickness $1 \mathrm{~mm}$ & Slice thickness $3 \mathrm{~mm}$ \\
\hline
\end{tabular}

\subsection{Unity game engine and visualisation}

The meshed colons and coordinates of the centreline were imported into the Unity game engine v5.1. Unity is an effective development environment for Oculus Rift applications and inherently supports virtual reality visualisation and interaction. Two rendered versions of the colon were imported - one with the normal vectors facing inward the other with them pointing outwards (allowing the colon to be visible from both inside and out). This allowed the user to gain an overall view of the colon from the outside by "flying" along an arbitrary path around its exterior before entering via the caecum. Once inside the colon the coordinates of the centreline were plotted using the iTween plugin for Unity [Pixel Placement, Baltimore, Maryland, USA] and the virtual reality camera assigned to travel along the centreline coordinates. A point light source was used to light the mucosal surface and was assigned to track the camera, maintaining a constant distance beneath it throughout the colonoscopy.

The camera was assigned to automatically travel at a set speed through the colon but its positon could be controlled using an Xbox gaming controller with the following assigned functionalities:

- The "A" button paused and resumed the automatic movement of the camera along the centreline at its predefined speed.

- The up/down keys on the directional pad (D-pad) moved the camera forwards and backwards along the centreline in the colon. Usage of these buttons auto- matically overrides the automatic movement of the camera.

- The "B" button allowed the user to view their current position within the colon. It paused the movement of the camera inside the colon and switched to a stationary camera positioned above the colon. When viewing from the second, external camera the position of the user inside the colon was highlighted by a visible red dot. A second tap of the "B" button returned the view to the camera in the colon and resumed its motion.

The speed at which the camera travelled through the colon was carefully chosen to limit the chance of inducing nausea. During the development phase the transit speed was reduced incrementally and tested on an operator known to be sensitive to simulation sickness. The maximum speed at which the operator experienced a complete absence of nausea was selected. At the speed selected a complete, uninterrupted transit of study 1 takes 2 minutes and study 2, 4 minutes.

Camera orientation was controlled entirely by the movement of the user's head using the Oculus Rift head tracker. The Oculus Rift DK2 was connected to a middle to high-end desktop PC (Intel i7 4770k 3.5GHz, NVidia GeForce GTX 770, Windows 7) with no noticeable latency in the image. The observer was confronted with an external view of the colon when first wearing the Oculus Rift, providing an external flyby before briefly coming to a halt at the entrance to the caecum. The observer was then either automatically moved through the anatomy or, by utilising the Xbox controller, could proceed at their own speed whilst exploring the views via natural head movements.

\section{RESUltS}

Our developments have permitted stereoscopic 3D exploration of the interior surface of the colon both from the inside and out by utilisation of the Oculus Rift and Unity game engine. The experimental setup and example views from inside and outside the colon are shown in Figures 2-4.

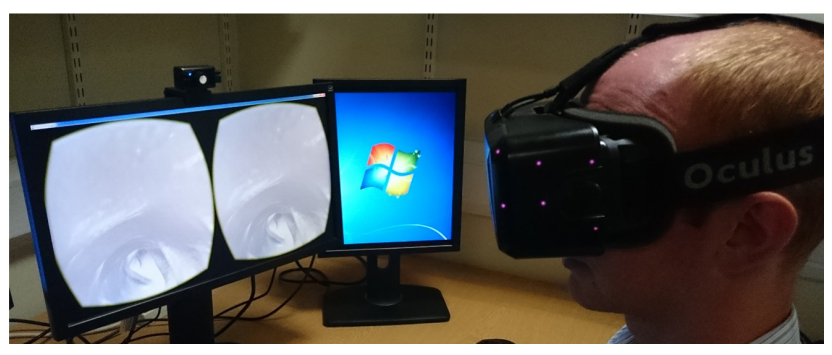

Figure 2. Schematic of the process used to produce an Oculus Rift virtual colonoscopy (ORVC) 


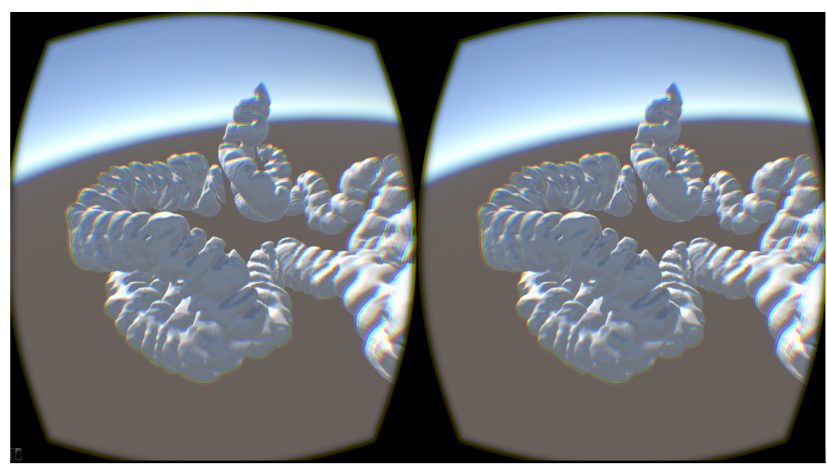

Figure 3. Oculus Rift DK2 stereoscopic view of the colon exterior of Study 2

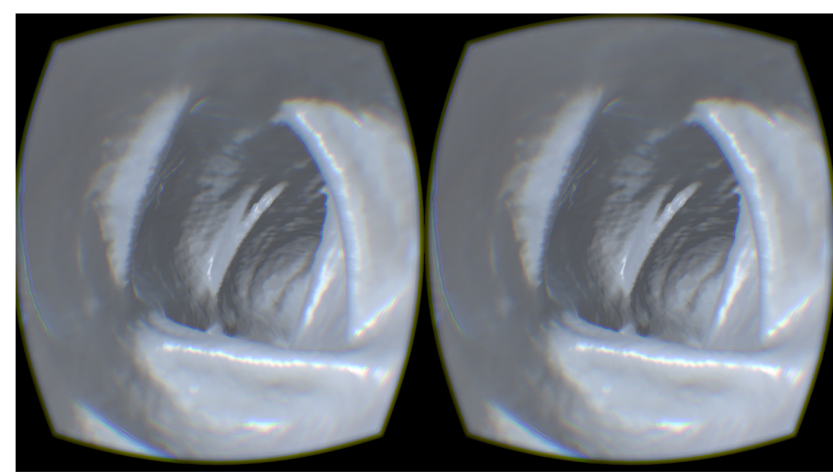

Figure 4. Oculus Rift DK2 stereoscopic view during the virtual colonoscopy of Study 1

Both the ORVCs studies were reviewed by three experienced clinicians all familiar with virtual colonoscopies: two radiologists and a gastroenterologist. All three were asked to provide a reflective statement on the experience and to draw comparisons to conventional virtual colonoscopy and optical colonoscopy. The comments are summarised in bullet point form below:

\section{Radiologist 1}

(1) Generally: Gained an immediate impression of the great potential of the technique. It offers a number of potential improvements on the conventional virtual colonoscopy fly-through technique but certain refinements are necessary for the ORVC technique to replace conventional virtual colonoscopy.

(2) The immersion and stereoscopic vision presents a strong possibility for increased sensitivity and specificity. A greater effect may be expected in less experienced radiologists who have yet to become overly familiar with the conventional virtual colonoscopy and have not developed a refined routine for their reporting. For radiologists exposed to the ORVC as standard training the rewards are likely to be even greater.
(3) Currently no more than four virtual colonoscopies would be reported in one morning/day due to fatigue in their labour intensive reporting. The ORVC felt comfortable and presents a possibility of reducing fatigue.

(4) An experienced virtual colonoscopy radiologist takes approximately 20 minutes per examination whereas an inexperienced radiologist may take in excess of 30 minutes. With appropriate training and experience with the ORVC, a reduction in examination time is a plausible prospect, particularly for less experienced radiologists.

(5) For the ORVC to be viable, an option for the camera to be orientated with the direction of travel is a necessity.

(6) When reporting on current systems a raft of supplementary information is available to the radiologist. This information would be useful if incorporated into the ORVC, in particular: indication of the direction of travel, information on current location in relation to the CT slices and the ability to mark suspicious areas on the fly-through and triangulate their positions on the CT image planes.

\section{Radiologist 2}

(1) Generally: The prospect of incorporating virtual reality imaging into diagnostics is an exciting potential development that would significantly impact on the way radiologists work. It removes the demanding process of assimilating a large data set.

(2) The Oculus Rift's intuitive head tracking ability provides the opportunity of looking around the haustral folds of the colon simply by turning one's head rather than complex movements with the mouse and screen.

(3) Immersion within the data removes the potential for error generated by distractions.

(4) The strong visual stimulus provided by the Oculus Rift means the generation of motion sickness is a real possibility.

(5) When reporting, the virtual colonoscopy cannot be utilised on its own, several pieces of information are considered in order to make diagnostic decisions. The CT slices contain information of structures outside the colon and this cannot be ignored. A primary tool used during reporting is the marking of features in the virtual colonoscopy and subsequently matching these to the CT slices and vice-versa. Such a feature would need to be incorporated into the ORVC technique for its clinical implementation. Routine reporting of the rest of the abdomen and pelvis would still be required.

(6) The psychophysiology of perception is complex and this new way of generating and looking at images 
needs to be assessed. This work gives motivation for comparisons of sensitivities and specificities with a current "gold standard". This could be optical colonoscopy but a comparison with CT colonography would be equally valid.

\section{Gastroenterologist}

(1) In optical colonoscopy the colonoscopist drives forward from anal canal to caecum. The gradual withdrawal of the instrument from the caecum allows the reverse view, likened to that seen in the wing mirrors of a car. The colon is a haustral structure: hence a polyp within a recess may be missed when driving toward the caecum but detected in the "wing mirrors" while exiting. Virtual colonoscopy offers a similar view with the advantage of driving forwards in both directions.

(2) The wider field of view offered in the ORVC is far greater than that offered by optical colonoscopy. Selfevidently this is of potential benefit for detecting lesions, which in turn increases confidence that fewer lesions might have been missed.

(3) Oculus Rift assisted virtual colonoscopy, by its very nature, offers unique and major advantages. In standard virtual colonoscopy, driving is guided by a mouse/keyboard control which is often "jerky" and does not readily afford fine changes. In contrast ORVC offers a refined natural method of viewing by slight movements of the head and changes in body position.

(4) The ORVC technique implemented allows a birdseye view of the inner wall of the colon, but crucially, viewed from the exterior. Consequently polyps located in haustral recesses are more readily identified, particularly at areas of natural angulation of the colon. The same view also allows identification of diverticulae. Colonic diverticulae are mucosal herniations through its confining wall, hence are more prominent when viewed from the outside.

(5) ORVC offers a different and unique advantage, that of total immersion within the environment, supplemented by looking at that same environment from the outside i.e. being both within the data and then viewing it from outwith. Put differently this can be likened to looking at the fish tank from the inside and outside.

The two radiologists independently shared several similar views while the gastroenterologist also had some overlapping points. All parties commented that full examination of the first colon (i.e. high resolution) had identified all lesions: polyp and diverticulum, and were confident that no other abnormality had been missed. An example of a diverticulum viewed on the Oculus Rift (from both inside and outside) is shown in Figure 5.

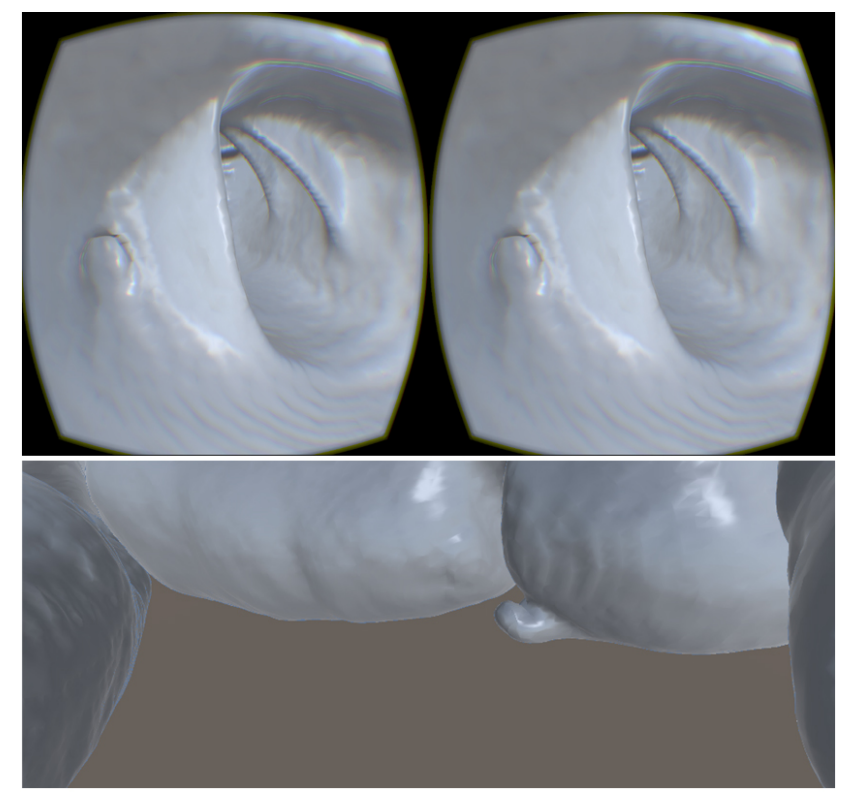

Figure 5. Both inside and outside views of a diverticulum spotted during examinations of Study 1

\section{Discussion}

Optical colonoscopy is widely considered the most sensitive diagnostic procedure for the detection of colonic pathologies. ${ }^{[14,15]}$ There is however growing evidence that virtual colonoscopy is improving towards similar sensitivity values, ${ }^{[3,16]}$ this is reflected by several national guidelines endorsing its use. ${ }^{[2,17]}$ With wider uptake encouraged, the numbers undergoing virtual colonoscopy will steadily rise. It is therefore important to optimise the technique for both lesion detectability and examination time.

Current practice of reporting CT uses sequential, thin 2dimensional slices, which are projected in 3 different planes (axial, sagittal and coronal). Alternating between the 3 different planes and moving backwards and forwards within them, the reporting radiologist will create a "3D" image in the mind. This is a complex process which is time consuming, requires experience, and involves the mental processing of large amounts of information whilst physically scrolling with the mouse on different screens. Although current software will allow 3D reconstructions for further interrogation, these too are displayed on the flat screen. The innovative addition of virtual reality potentially by-passes much of the mental processing and mechanical scrolling and presents the radiologist with a "simple" image for interpretation.

A wide range of immersive virtual reality headset technologies have entered the market. Figure 6 ranks the major virtual 
reality headsets (both those available now and those soon to be released) according to their respective prices at time of writing (November 2015).

All headsets listed in Figure 6 offer stereoscopic vision and head tracking capabilities but each offers slight variations and unique selling points such as customisability, cost, comfort and compatibility/integration with software. The Google Cardboard was tested using a variety of smartphones and the lack of processing power led to framerate limitations that were judged to be too debilitating for the virtual colonoscopy application. This issue was expected to apply to other smartphone-based headsets (all those with blue columns in Figure 6) and therefore these were not considered. Of the dedicated virtual reality headsets FOVE VR is of particular interest. It has not yet been released but offers the intriguing addition of built in eye tracking that makes it a consideration for future applications. Other high profile headsets not included in Figure 6 are Playstation VR and the Avegant Glyph. Playstation VR has not yet been released or associated with a price and is likely to be heavily geared toward development for the PlayStation game console platform - it was therefore not considered. The Avegant Glyph is a different implementation, employing retinal projection technology rather than OLED (organic light-emitting diode) screens. The projectors are embedded into a portable visor/headphone device primarily designed to project media from connected devices (such as smartphones) directly to the eye for an enhanced display. At present the Oculus Rift DK2 headset offered the best prospect for our application due to its more advanced stage of development, extensive community support, competitive price and integration with the Unity game engine. The commercialised version of the Oculus Rift has yet to be released but applications developed for the DK2 are expected to be easily transferable to the commercial version.

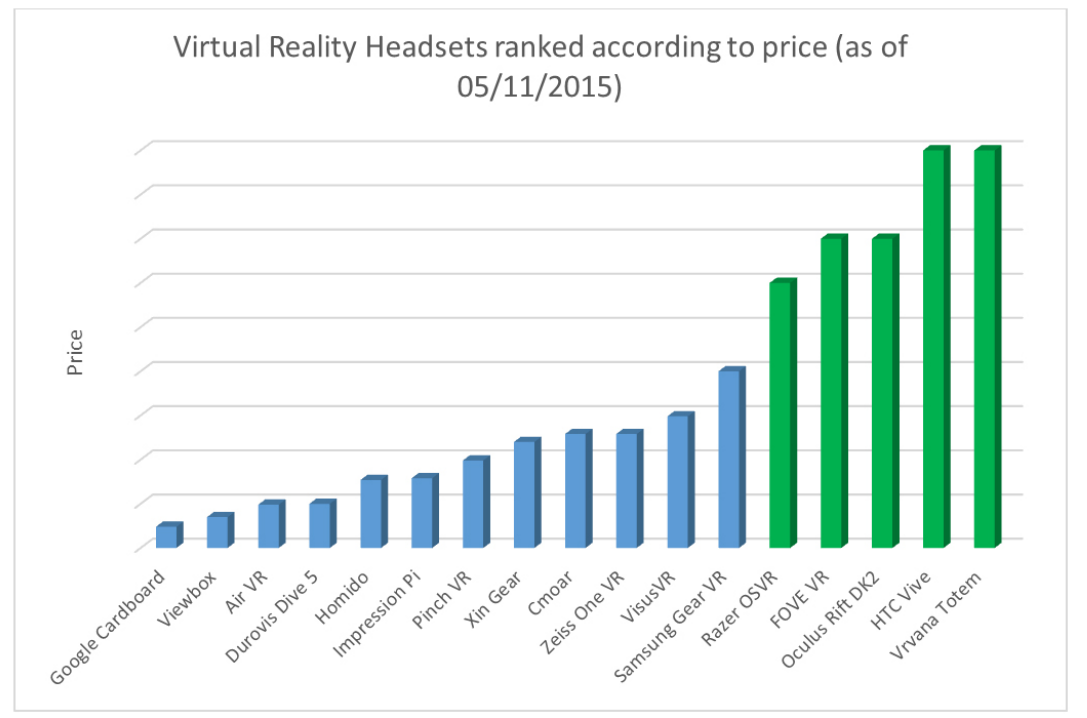

Figure 6. List of virtual reality devices ranked according to price. Columns in blue indicate devices which require a smartphone, columns in green indicate dedicated virtual reality headset devices. Prices of some devices not yet released are speculative at time of writing $(05 / 11 / 2015)$
The Oculus Rift DK2 performed very smoothly in the tests and the high specification PC and graphics card ensured lag free and artefact free viewing. The Unity game engine provided the flexibility for rapid development of the environment and accommodation of the Xbox game controller. Although application development within Unity involves a fairly steep learning curve for the uninitiated, the breadth of online resources helps considerably. At our current stage we are able to go from raw $\mathrm{CT}$ data to visualisation in the $\mathrm{Ocu}-$ lus Rift within approximately 2 hours (without significant complications) and this is likely to reduce with increased experience. Furthermore, improvements in all areas can be expected as the technology progresses.

The conventional method of viewing colonoscopies (on a

Published by Sciedu Press workstation monitor) presents some challenges. Firstly, the use of a mouse and keyboard, although familiar, is not an optimal user interface. Secondly, in colonoscopies the region of interest is the entire inner surface of the colon wall which is a large $3 \mathrm{D}$ surface area being represented by a series of $2 \mathrm{D}$ projections on a monitor. In order for a single projection to show the data effectively requires either a sufficiently wide angle view (e.g. fisheye-like lens) which distorts the image, or the user needs to be able to change the view quickly and efficiently while maintaining orientation - limited by the mouse/keyboard interface. Our pilot observations suggest the Oculus Rift offers a major improvement on these aspects:

- It presents the user with stereoscopic viewing ability and depth perception, potentially allowing for height- 
ened localisation/characterisation of protrusions or lumps such as polyps.

- The head tracking gives a more natural means for camera reorientation, which the clinicians found particularly useful when examining areas behind haustral folds.

The design chosen also allowed the colonic mucosa to be viewed from both its luminal aspect and from the outside, the two together giving a more complete view. This novel feature of the ORVC proved useful to the radiologists and the gastroenterologist in detecting abnormalities. Additionally, the Oculus Rift provides a fully immersive experience by providing the user with a sense of "presence within the data", rather than as a passive distant observer as is the case when using a mouse/keyboard and a monitor screen. This is likely to reduce distraction and heighten awareness of their virtual surroundings. Finally, when viewing from within the colon it is not always possible for the user to be aware of their precise location. Despite this being made possible by the external camera which rapidly provided the triangulation necessary, the comments made by the radiologists suggest a need for advancing this feature in order to place specific features into context with the original CT slices.

The resolution of the DK2 display is $960 \times 1080$ to each eye. The DK2 OLED display has a visible grid-like appearance which may be attributed to a poor pixel fill factor (no. of pixels per unit area) effectively producing small gaps between pixels. Generally speaking this feature is more noticeable on still scenes with smaller objects. During the ORVC examination this issue is less noticeable probably due to the user's attention being drawn to larger objects spanning across a large number of pixels. Also the post-processing of the CT images used to create the virtual colonoscopy effectively smooths the data. For these reasons it is unlikely that this affects the interpretation of the virtual colonoscopy. However, when considering viewing high resolution, unprocessed CT slices this is expected to adversely affect interpretation. With subsequent releases of the technology the resolution and pixel fill factor will improve and its effect on CT slice reporting should be assessed.

The variety in data quality of the two ORVC studies gave an appreciation of a range of appearances to be expected when using this technique. From our own experience and the clinicians' comments, a summarised collation of advantages and disadvantages relative to the conventional virtual colonoscopy are noted below:

- Advantages

(1) Immersive experience - more engaged with the data leading to improved awareness without distractions.

(2) More intuitively interactive - instead of moving a mouse to change camera direction the natural movement of the head changes the view.

(3) Stereoscopic vision to help detection of 3D undulations in the surface of the colon (e.g. polyps).

(4) Improved examination around colonic folds.

(5) No wide angle lens distortion as currently implemented in virtual colonoscopy software on $2 \mathrm{D}$ monitors.

(6) Less mentally intensive experience leading to a potential for fatigue reduction.

(7) View of the inside surface of the colon from the outside offers a potentially useful diagnostic angle.

- Drawbacks

(1) User must rotate their body/head up to $180^{\circ}$ adding an ergonomic complication and inconvenience.

(2) Some users can find the experience mildly nauseating due to the mismatch between visual and vestibular inputs (i.e. visually moving while remaining physically stationary).

(3) Lack of functionality to quickly and efficiently correlate findings between virtual colonoscopy and CT slices.

(4) The current resolution displayed to each eye by the Oculus Rift DK2 $(960 \times 1080$ pixels $)$ and the poor pixel fill factor (no of pixels per unit area), combined with the proximity of the eye, creates a pixelated appearance that is not present on a standard monitor. While potentially distracting with the DK2 this is unlikely to be an issue as virtual reality headset technology matures.

Solutions to these drawbacks are beyond the scope of this paper, but improvements such as the option to orientate the camera along the direction of movement (such as in a rollercoaster) and incorporation of CT slice visualisation in the virtual world, have been considered and will form the basis of future work. The success of viewing the colon from the outside introduces another focus for further development. Currently the user is presented with a series of arbitrary, preset viewing positions but given the feedback, there is merit in offering a greater range of viewing angles and giving the user more positional control.

Implementation of the future developments above may also enhance the ability to stage cancer. Firstly, the ability to review the original CT data is important to help determine the 
extent/spread of the lesion. Secondly, additional information could be presented to the radiologist via segmentation and external observation of the outer lining of the colon. Such a view could highlight prominent undulations in the external surface of the colon to help determine whether the cancer is of an early stage (T1, T2 - contained within the inner layers of the bowel wall) or later stage (T3, T4 - penetrated the outer layers of the bowel wall). However, segmentation of the outer wall of the colon is likely to pose significant challenges due to the reduced contrast between the colon and surrounding tissue compared to its gas-filled lumen. Nonetheless this remains a consideration for the future.

At this early stage it is difficult to comment on any improvement in examination time offered relative to the conventional virtual colonoscopy. However, with the ease of camera orientation offered by the head tracking capability of the Oculus Rift and as more experience is acquired, a decrease in examination time is conceivable. The transit speed through the lumen of the colon is ultimately limited by the operator's susceptibility to nausea from simulation sickness. For each of the two studies presented the transit speed was optimised for a single operator known to be sensitive to simulation sickness with the expectation that this speed was comfortable for most other operators. This proved to be the case as none of the clinical operators experienced nausea during the examinations. The nauseating effects are further reduced with the functionality of controlling the position using the D-pad on the Xbox controller. This feature may be optimised further by allowing the user to change the transit speed and sensitivity of the D-pad buttons.

This preliminary work has highlighted the benefits and challenges of virtual colonoscopy in an immersive environment and indicated further steps that are necessary to make the
ORVC a more viable diagnostic technique. Upon completion of the development work the trajectory is toward a prospective pilot study to compare conventional and Oculus Rift virtual colonoscopies. Assessment of this new technique with the use of the Receiver Operative Curve (ROC) would help define its place in the rapidly developing world of diagnostic technology.

\section{Conclusion}

A mechanism for viewing virtual colonoscopies with an Oculus Rift has been successfully implemented and trialled on three clinicians. Initial testing has produced promising reactions from clinical colleagues and confirms the potential of this novel visualisation method. It has been acknowledged that several improvements are necessary before this technique is likely to be of significant clinical benefit but early potential has been demonstrated. Overall, the feedback is one of support, providing encouragement for pursuing this work further. After incorporation of the necessary developments, the next steps will involve a semi-quantitative pilot study which will aim to compare clinicians' experiences and reporting confidence with ORVC and traditional virtual colonoscopy.

\section{ACKNOWLEDGements}

The authors would like to thank The Bardhan Research and Education Trust of Rotherham (BRET) for supporting this research. We would also like to acknowledge TeraRecon for giving permission for use of their data and Tzen S Toh, Ayesha A Girach and Samuel Birch-Machin from The University of Sheffield for their contributions.

\section{CONFlicts OF INTEREST Disclosure}

The authors declare that they have no competing interests.

\section{REFERENCES}

[1] Bowel Cancer Mortality Statistics [Internet]. London: Cancer Research UK; c2002-2015 [cited 2015 Sept 12]. Available from: http://www . cancerresearchuk. org/health-professiona 1/cancer-statistics/statistics-by-cancer-type/bowe 1-cancer/mortality\#heading-Two

[2] Levin B, Lieberman DA, McFarland B, et al. Screening and Surveillance for the Early Detection of Colorectal Cancer and Adenomatous Polyps, 2008: A Joint Guideline From the American Cancer Society, the US Multi-Society Task Force on Colorectal Cancer, and the American College of Radiology. Gastroenterology. 2008; 134: 1570-95. PMid: 18384785. http://dx.doi.org/10.1053/j.gastro 20 08.02 .002

[3] Pickhardt PJ, Hassan C, Halligan S, et al. Colorectal Cancer: CT Colonography and Colonoscopy for Detection - Systematic Re- view and Meta-Analysis. Radiology. 2011; 259: 393-405. http: //dx.doi.org/10.1148/radiol.11101887

[4] Halligan S, Altman DG, Taylor SA, et al. CT Colonography in the Detection of Colorectal Polyps and Cancer: Systematic Review, Meta-Analysis, and Proposed Minimum Data Set for Study Level Reporting. Radiology. 2005; 237: 893-904. http://dx.doi.org /10.1148/radiol. 2373050176

[5] d. Haan MC, v. Gelder RE, Graser A, et al. Diagnostic value of CT-colonography as compared to colonoscopy in an asymptomatic screening population: a meta-analysis. European Radiology. 2011; 21: 1747-63. http://dx.doi.org/10.1007/s00330-011-210 4-8

[6] Barish MA, Soto JA, Ferrucci JT. Consensus on current clinical practice of virtual colonoscopy. Am J Roentgenol. 2005; 184: 786-792. PMid: 15728598. http://dx.doi.org/10.2214/ajr.184.3.0 1840786 
[7] Ball S, Bluteau P, Clouder L, et al. myShoes: An Immersive Simulation of Dementia. Proceedings of the 10th International Conference on E-Learning (ICEL 2015); 2015 June 25-26; Nassau, Bahamas. Sonning Common, UK: Academic Conferences and Publishing International Limited; 2015.

[8] Chiarovano E, de Waele C, MacDougall HG, et al. Maintaining Balance when Looking at a Virtual Reality Three-Dimensional Display of a Field of Moving Dots or at a Virtual Reality Scene. Frontiers in Neurology. 2015; 6: 164. PMid: 26284023. http: //dx.doi.org/10.3389/fneur.2015.00164

[9] Fulvio J, Wang M, Rokers B. Head tracking in virtual reality displays reduces the misperception of 3D motion. Journal of Vision. 2015; 15 1180. PMid: 26326868. http://dx.doi.org/10.1167/15.12.1 180

[10] Hoffman HG, Meyer WJ, Ramirez M, et al. Feasibility of Articulated Arm Mounted Oculus Rift Virtual Reality Goggles for Adjunctive Pain Control During Occupational Therapy in Pediatric Burn Patients. Cyberpsychology Behavior and Social Netweorking. 2014; 17: $397-$ 401. PMid: 24892204. http://dx.doi.org/10.1089/cyber. 2 014.0058

[11] Kim J, Chung CYL, Nakamura S, et al. The Oculus Rift: a costeffective tool for studying visual-vestibular interactions in selfmotion perception. Frontiers in Psychology. 2015; 6: 248. PMid: 25821438. http://dx.doi.org/10.3389/fpsyg. 2015.00248
[12] Xu X, Chen KB, Lin J, et al. The accuracy of the Oculus Rift virtual reality head-mounted display during cervical spine mobility measurement. Journal of Biomechanics. 2015; 48: 721-4. PMid: 25636855. http://dx.doi.org/10.1016/j.jbiomech.2015.01.005

[13] Mirhosseini K, Sun Q, Gurijala KC, et al. Benefits of 3D immersion for virtual colonoscopy. Proceedings of: 3DVis, 2014 IEEE VIS International Workshop; 2014 Nov 9; Paris, France. IEEE. 2014. 75-79 p. http://dx.doi.org/10.1109/3DVis.2014.7160105

[14] Asfaw A, Gustafsson-Wright E, Vander Gaag J. Willingness to pay for health insurance: An analysis of the potential market for new low-cost health insurance products in Namibia, Amsterdam Institute for International Development. 2008; 1-22. http://dx.doi.org /10.1016/j.amjmed.2006.05.061

[15] Lieberman D. Progress and Challenges in Colorectal Cancer Screening and Surveillance. Gastroenterology. 2010; 138: 2115-26. http: //dx.doi.org/10.1053/j.gastro.2010.02.006

[16] Graser A, Stieber P, Nagel D, et al. Comparison of CT colonography, colonoscopy, sigmoidoscopy and faecal occult blood tests for the detection of advanced adenoma in an average risk population. Gut. 2009; 58: 241-8. http://dx.doi.org/10.1136/gut. 2008.156448

[17] Chapman K, editor. Guidelines for the use of imaging in the NHS Bowel Cancer Screening Programme, second edition. NHS Cancer Screening Programmes; 2012 Nov. 